\title{
LA VITESSE DE REMONTÉE DES SPERMATOZOÏDES DANS LE TRACTUS GÉNITAL DE LA BREBIS
}

PAR

\section{DAUZIER et Suzanne WINTENBERGER}

Station de Physiologie animale du Centre National de Recherches Zootechniques, Jouy-en-Josas $\left({ }^{1}\right)$

La durée de vie de l'œuf tubaire des Mammifères étant courte, il importe que des spermatozoïdes vivants se trouvent dans la partie terminale de la trompe au moment où l'œuf est pondu. Pour que cette condition puisse être réalisée, il est indispensable de connaître, pour chaque espèce, le temps nécessaire à la remontée des gamètes mâles depuis le vagin jusqu'au pavillon, afin de fixer avec précision l'heure la plus favorable pour l'accouplement ou pour l'insémination. Cette connaissance peut donc permettre une amélioration sensible du taux de fécondation, c'est pourquoi elle a donné lieu à de nombreux travaux. Malheureusement les résultats de ces recherches se contredisent et, il n'a pas été possible jusqu'ici d'en tirer parti dans la pratique de l'élevage.

C'est ainsi que chez la Brebis, Quinian, Maré et Roux (I932), GreEN et WINTERS (I935), KELLEY (I937) ont évalué la durée de remontée à un minimum de 5 heures, tandis que Schotr et PHILLIPS (I94I), au contraire, estiment que dans la plupart des cas les spermatozoïdes atteignent le pavillon en moins de 20 minutes. STARKE (I949) approuve les conclusions de ces auteurs et admet qu'à partir de 6 minutes il est possible de retrouver des spermatozoïdes dans la partie terminale des trompes.

On relève des contradictions analogues dans les résultats obtenus avec d'autres espèces. Skatkine et Roumiantzewa (I948) affirment que, chez la lapine, les premiers spermatozoïdes atteignent l'oviducte 6 à 8 heures après l'accouplement, alors que HENSEN ( 1876 ) avait trouvé des spermatozoïdes dans la partie supérieure des trompes de Fallope, 2 h $25 \mathrm{~m}$. après le coït.

C'est pourquoi nous avons pensé que de nouvelles observations s'imposaient afin d'apporter une réponse sûre à cette importante question.

(1) Travaux réalisés à la Bergerie nationale de Rambouillet et au Laboratoire d'Évoution des Etres organisés. 


\section{MATERIEL ET TECHNIQUE}

Nos recherches ont été réalisées avec 62 Brebis :

37 Ile-de-France, I4 Solognotes, 6 Berrichonnes du Cher, 5 Mérinos de Rambouillet.

L'œstrus était détecté matin, midi et soir. Des Béliers berrichons furent utilisés pour les accouplements, et la qualité du sperme régulièrement contrôlée. En outre, après l'accouplement, par prélèvement dans le vagin, on évaluait la proportion de spermatozoïdes anormaux et la motilité relative. Les accouplements ou les récoltes de sperme pour le contrôle furent effectués régulièrement tous les 2 ou 3 jours environ.

Le prélèvement du tractus génital en vue de la recherche des spermatozoïdes fut effectué immédiatement après l'égorgement en prenant toutes les précautions désirables pour éviter le transport accidentel de spermatozoïdes, par contact direct ou par les instruments, dans les régions n'en contenant pas ou peu.

Nous avons choisi la technique des frottis de préférence à celle de la perfusion, car elle permet, pour des régions contenant relativement peu de spermatozoïdes (isthme et ampoule), d'obtenir le nombre total de spermatozoïdes par unité de longueur. En effet, en maintenant un fragment des trompes de Fallope $(2 \mathrm{~mm})$ par une extrémité et en pressant progressivement de la partie maintenue vers la partie libre, on arrive à expulser non seulement le contenu de la lumière, mais encore tout l'épithélium tubaire interne ; l'ensemble est étalé sur une lame, fixé à l'alcool et coloré au rose bengale. Ainsi aucun spermatozoïde présent dans cette portion ne peut échapper à la numération.

Pour les pavillons, les cornes utérines et 1'utérus, nous avons prélevé une surface constante de ces organes, en effectuant trois frottis successifs avec le même fragment pour obtenir une valeur exacte du nombre de spermatozoïdes présents. Comme la $3^{\mathrm{e}}$ lame ne contenait jamais plus de I $6 \%$ (de o à I6\%; valeur moyenne : 7,4 ; écart-type : 4,5), du nombre total des spermatozoïdes recueillis sur les 3 lames, nous avons conclu, d'une part, que la technique du prélèvement par frottis, pour les organes mentionnés, permettait d'obtenir une valeur exacte du nombre de spermatozoïdes présents, et d'autre part, qu'il était pratiquement suffisant d'effectuer deux frottis pour chaque prélèvement. Dans les tableaux ci-joints nous avons fait figurer un seul nombre, il représente la somme des deux premières lames.

\section{VITESSE DE REMONTEE DES SPERMATOZOIDES}

27 Brebis ont été accouplées dès l'apparition des chaleurs, et 35 autres vers la fin de celles-ci.

Les résultats obtenus en début de rut, sont présentés dans le tableau I, quand l'intervalle entre le coit et le prélèvement est inférieur ou égal à une heure, et dans le tableau II pour les intervalles supérieurs. 
On constate que les spermatozoïdes sont parvenus au milieu du col de l'utérus I5 minutes après 1'accouplement et ont franchi le cervix en 30 minutes. Le sommet des cornes utérines est atteint au bout de 2 heures chez 3 Brebis sur 4, et de une heure dans un seul cas.

La remontée des spermatozoïdes dans les oviductes est plus lente, on en trouve au milieu de l'isthme à partir de 6 heures et au milieu de l'ampoule à partir de 8 heures.

\section{TABLEAU I}

\begin{tabular}{|c|c|c|c|c|c|c|c|c|c|c|c|c|}
\hline \multirow{2}{*}{$\begin{array}{c}\text { Brebis } \\
\text { numéro }\end{array}$} & \multirow{2}{*}{ Rare } & \multirow{2}{*}{1} & \multirow{2}{*}{$\mathrm{Ce}$} & \multicolumn{3}{|c|}{ Cervix } & \multirow{2}{*}{ Utérus } & \multirow{2}{*}{$C_{3}$} & \multirow{2}{*}{$\mathrm{C}_{2}$} & \multirow{2}{*}{$\mathrm{C}_{\mathrm{I}}$} & \multirow{2}{*}{ B. I. } & \multirow{2}{*}{ Côté } \\
\hline & & & & entrée & milieu & sortie & & & & & & \\
\hline \multirow{2}{*}{126} & \multirow{2}{*}{$\begin{array}{l}\text { Ile-de- } \\
\text { France }\end{array}$} & \multirow{2}{*}{$15 \mathrm{~m}$} & \multirow{2}{*}{++} & \multirow[t]{2}{*}{$\infty$} & \multirow{2}{*}{2500} & \multirow{2}{*}{0} & \multirow{2}{*}{0} & $\circ$ & $\stackrel{\circ}{0}$ & $\circ$ & o & Gauche \\
\hline & & & & & & & & 0 & $\circ$ & ० & 0 & Droit \\
\hline \multirow{2}{*}{135} & \multirow{2}{*}{$\begin{array}{l}\text { Ile-de- } \\
\text { France }\end{array}$} & \multirow{2}{*}{$15 \mathrm{~m}$} & \multirow{2}{*}{$+t$} & \multirow{2}{*}{$\infty$} & \multirow{2}{*}{ I } & \multirow{2}{*}{$\circ$} & \multirow{2}{*}{0} & 0 & $\circ$ & $\circ$ & 0 & Gauche \\
\hline & & & & & & & & 0 & o & 0 & 0 & Droit \\
\hline \multirow{2}{*}{2} & \multirow{2}{*}{$\begin{array}{l}\text { Berri- } \\
\text { chonne }\end{array}$} & \multirow{2}{*}{$15 \mathrm{~m}$} & \multirow{2}{*}{ t } & \multirow{2}{*}{$\infty$} & \multirow{2}{*}{ I } & 0 & 0 & o & 0 & 。 & o & Gauche \\
\hline & & & & & & & & 0 & 0 & 。 & ○ & Droit \\
\hline 143 & Ile-de- & $30 \mathrm{~m}$ & ++ & $\infty$ & & $\infty$ & 832 & I & $\circ$ & $\circ$ & $\circ$ & Gauche \\
\hline & France & & & & & & & 5 & 0 & o & o & Droit \\
\hline I 400 bis & Solo- & $30 \mathrm{~m}$ & ++ & $\infty$ & & 304 & I & 0 & 은 & o & o & Gauche \\
\hline & gnole & & & & & & & I & 0 & o & o & Droit \\
\hline & Berri- & & & & & & & $\circ$ & o & $\circ$ & $\circ$ & Gauche \\
\hline 020 & chonne & 3011 & 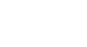 & 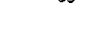 & & 100 & 0 & o & o & 0 & 0 & Droit \\
\hline 97 & Ile-de- & 60 & 1 & $\infty$ & & 0 & 0 & o & o & o & $\circ$ & Gauche \\
\hline (3) & France & & & & & & & 0 & o & o & o & Droit \\
\hline 1052 & Solo- & 60 & 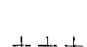 & $\infty$ & & & & 34 & I9 & 22 & I & Gauche \\
\hline-0 & gnote & 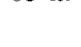 & 1 & & & 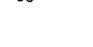 & 407 & 96 & $3 \mathrm{I}$ & $6 \mathrm{I}$ & $\circ$ & Droit \\
\hline & & & & $m$ & & & & 0 & o & o & o & Gauche \\
\hline $1 / 3$ & France & . & T & 然 & & 5 & 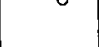 & 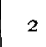 & $\circ$ & 0 & o & Droit \\
\hline
\end{tabular}

$I=$ Intervalle entre l'accouplement et le prélèvement du tractus génital.

$\mathrm{C}_{e}=$ État du Cervix.

$+t+$ Cervix très ouvert.

$\mathrm{C}_{\mathbf{3}}=$ Base des cornes utérines.

$+t$ Cervix ouvert.

$+\quad$ Cervix peu ouvert.

$\mathrm{C}_{2}=$ Milieu des cornes utérines.

- Cervix fermé.

$\mathrm{C}_{1}=$ Sommet des cornes utérines.

B. I. = Base de !'isthme. 


\section{TABLEAU II}

\begin{tabular}{|c|c|c|c|c|c|c|c|c|c|c|}
\hline $\begin{array}{c}\text { Brebis } \\
\text { numéro }\end{array}$ & Race & I & Utérus & $\mathrm{C}_{3}$ & $\mathrm{C}_{2}$ & $\mathrm{C}_{1}$ & $\mathrm{~A}_{2}$ & $A_{1}$ & $\mathrm{P}$ & Côté \\
\hline \multirow{2}{*}{ I 421} & \multirow{2}{*}{ Solognote } & \multirow{2}{*}{$2 \mathrm{~h}$} & \multirow{2}{*}{ 6I } & 535 & 258 & 5993 & 0 & 0 & $\circ$ & Gauche \\
\hline & & & & ${ }^{15} \mathrm{I}$ & 307 & 2630 & o & 0 & 0 & Droit \\
\hline \multirow{2}{*}{ I 4 II } & \multirow{2}{*}{ Solognote } & \multirow{2}{*}{$2 \mathrm{~h}$} & \multirow{2}{*}{$27^{8} 3$} & 0 & 0 & $\circ$ & 0 & 0 & 0 & Gauche \\
\hline & & & & $\circ$ & $\circ$ & $\circ$ & $\circ$ & $\circ$ & 0 & Droit \\
\hline \multirow{2}{*}{ I70 } & \multirow{2}{*}{$\begin{array}{l}\text { Ile-de- } \\
\text { France }\end{array}$} & \multirow{2}{*}{$2 \mathrm{~h}$} & \multirow{2}{*}{$2 \mathrm{I}$} & $\circ$ & $\circ$ & 6 & o & 0 & $\circ$ & Gauche \\
\hline & & & & $\mathrm{I}$ & $\circ$ & $\circ$ & $\circ$ & 0 & $\circ$ & Droit \\
\hline \multirow[b]{2}{*}{ 107 } & \multirow{2}{*}{$\begin{array}{l}\text { Ile-de- } \\
\text { France }\end{array}$} & \multirow{2}{*}{$2 \mathrm{~h}$} & \multirow{2}{*}{ IO } & $\mathrm{I}$ & $\circ$ & 2 & 0 & $\circ$ & $\circ$ & Gauche \\
\hline & & & & 0 & $\circ$ & $\bar{I}$ & 0 & 0 & $\circ$ & Droit \\
\hline \multirow{2}{*}{ I 427} & \multirow{2}{*}{ Solognote } & \multirow{2}{*}{$6 \mathrm{~h}$} & \multirow{2}{*}{ II 7} & 39 & 3 & 70 & 0 & $\circ$ & $\circ$ & Gauche \\
\hline & & & & 9 & I 50 & 337 & $\circ$ & 0 & 0 & Droit \\
\hline \multirow{2}{*}{68} & \multirow{2}{*}{$\begin{array}{l}\text { Berri- } \\
\text { chonne }\end{array}$} & & & 3 & I3 & 12 & 3 & 0 & 0 & Gauche \\
\hline & & $6 \mathrm{~h}$ & 68 & 25 & 39 & I78 & 0 & 0 & $\circ$ & Droit \\
\hline$J 100$ & Solognote & & & 253 & $5^{64}$ & 1006 & 0 & $\circ$ & $\therefore$ & Gauche \\
\hline I 409 & Solognote & $6 \mathrm{~h}$ & 3000 & I 680 & 4300 & $\infty$ & 2 & $\circ$ & 0 & Droit \\
\hline & Solognote & $6 \mathrm{~h}$ & 160 & 62 & 170 & 258 & 13 & o & 0 & Gauche \\
\hline I 4 OI & Solognote & $6 \mathrm{~h}$ & 160 & 43 & 185 & I 200 & 3 & $\circ$ & $\circ$ & Droit \\
\hline 1062 & Solognote & $8 \mathrm{~h}$ & 230 & 439 & 872 & 495 & 2 & 0 & $\circ$ & Gauche \\
\hline & & & $23^{\circ}$ & 500 & 92 & 535 & $\mathrm{I}$ & o & $\circ$ & Droit \\
\hline 1057 & Solognote & $8 \mathrm{~h}$ & 280 & 24 & 52 & 500 & 3 & $\mathbf{I}$ & $\circ$ & Gauche \\
\hline 1057 & & 811 & 200 & 34 & II & 163 & $\circ$ & $\circ$ & 0 & Droit \\
\hline & & & & $233^{I}$ & I 806 & 2739 & 0 & 2 & 0 & Gauche \\
\hline I 4 I9 & Solognote & $9 \mathrm{~h}$ & $\infty$ & 513 & 202 & 924 & 2 & 0 & 0 & Droit \\
\hline & Ile-de- & $\mathrm{ab}$ & & 393 & 2097 & 630 & $\circ$ & o & 0 & Gauche \\
\hline${ }^{1} 3^{2}$ & France & $9 \mathrm{n}$ & & $\infty$ & I 842 & 5232 & 0 & $\circ$ & 0 & Droit \\
\hline & Ile-de- & & & 3 & $\circ$ & 2 & 6 & $\circ$ & 0 & Gauche \\
\hline $9^{I}$ & France & $9 \mathrm{~h}$ & I9 & 68 & $\mathrm{I} 2$ & 4 & 0 & 0 & 0 & Droit \\
\hline 86 & Ile-de- & $9 \mathrm{~h}$ & 231 & $I_{4}$ & 25 & 7 & $\circ$ & $\circ$ & 0 & Gauche \\
\hline 80 & France & $9 \mathrm{n}$ & $23^{1}$ & 59 & 27 & 4 & $\circ$ & ? & 0 & Droit \\
\hline & Ile-de- & $\mathrm{L} 2 \mathrm{~h}$ & $6 \mathrm{r}$ & $\bar{I}$ & 8 & $5^{8}$ & I & 0 & 0 & Gauche \\
\hline 125 & France & $\mathrm{I} 2 \mathrm{~h}$ & $6 \mathbf{r}$ & 5 & 2 & $3^{8}$ & 0 & $\circ$ & 0 & Droit \\
\hline I 34 bis & Berri- & I2 h & & $3^{6}$ & 70 & 16 & 0 & 0 & 0 & Gauche \\
\hline 134025 & chonne & $12 \mathrm{n}$ & & 54 & $3 \mathrm{I}$ & 20 & 0 & $\mathrm{I}$ & 0 & Droit \\
\hline I4I & Ile-de- & $13 \mathrm{~h}$ & & 299 & 8 & 62 & $\circ$ & 0 & 0 & Gauche \\
\hline I4I & France & ${ }^{1} 3 \mathrm{n}$ & & 59 & 26 & 204 & 2 & 0 & $\circ$ & Droit \\
\hline I 50 & Ile-de- & $\mathrm{I} 3 \mathrm{~h}$ & & 2 & 5 & 33 & $\circ$ & 0 & $\circ$ & Gauche \\
\hline 159 & France & $13 n$ & & 5 & I & 35 & 0 & 0 & 0 & Droit \\
\hline I06 & Ile-de- & I & & $I$ & 1803 & 2565 & 0 & $\circ$ & 0 & Gauche \\
\hline I00 & France & $I_{2}$ & I 835 & $37 \underline{5}$ & 585 & 685 & 0 & $\circ$ & 0 & Droit \\
\hline & Ile-de- & $\mathrm{I}_{4} \mathrm{~h}$ & 204 & $5 \mathrm{I}$ & 4 & 62 & I 7 & o & 0 & Gauche \\
\hline 124 & France & $\mathrm{I}_{4} \mathrm{~h}$ & 204 & I9 & 36 & 0 & 0 & 0 & 0 & Droit \\
\hline 188 & Ile-de- & $I \Delta+>$ & 550 & 109 & I 785 & & 9 & I & 0 & Gauche \\
\hline 100 & $\mathrm{Fr}$ & $14 \pi$ & $55^{\circ}$ & $3 \mathrm{I}$ & 230 & & 243 & 5 & I & Droit \\
\hline
\end{tabular}

$A_{1}=$ Milieu de l'isthme; $A_{2}=$ Milieu de l'ampoule ; $P=$ Pavillon. 
Des vitesses de remontée plus grandes, du moins dans la partie cervicoutérine, ont été obtenues avec les Brebis accouplées vers la fin des chaleurs. En effet, le cervix est franchi en 30 minutes et, dans un cas sur trois, il l'est même $I_{5}$ minutes après le coït. Les spermatozoïdes sont présents au sommet des cornes utérines, chez 2 des 6 Brebis abattues, 30 minute (une) et une heure (''autre) après l'accouplement; mais ils n'atteignent jamais le milieu de l'isthme avant 4 heures et le pavillon avant 9 heures.

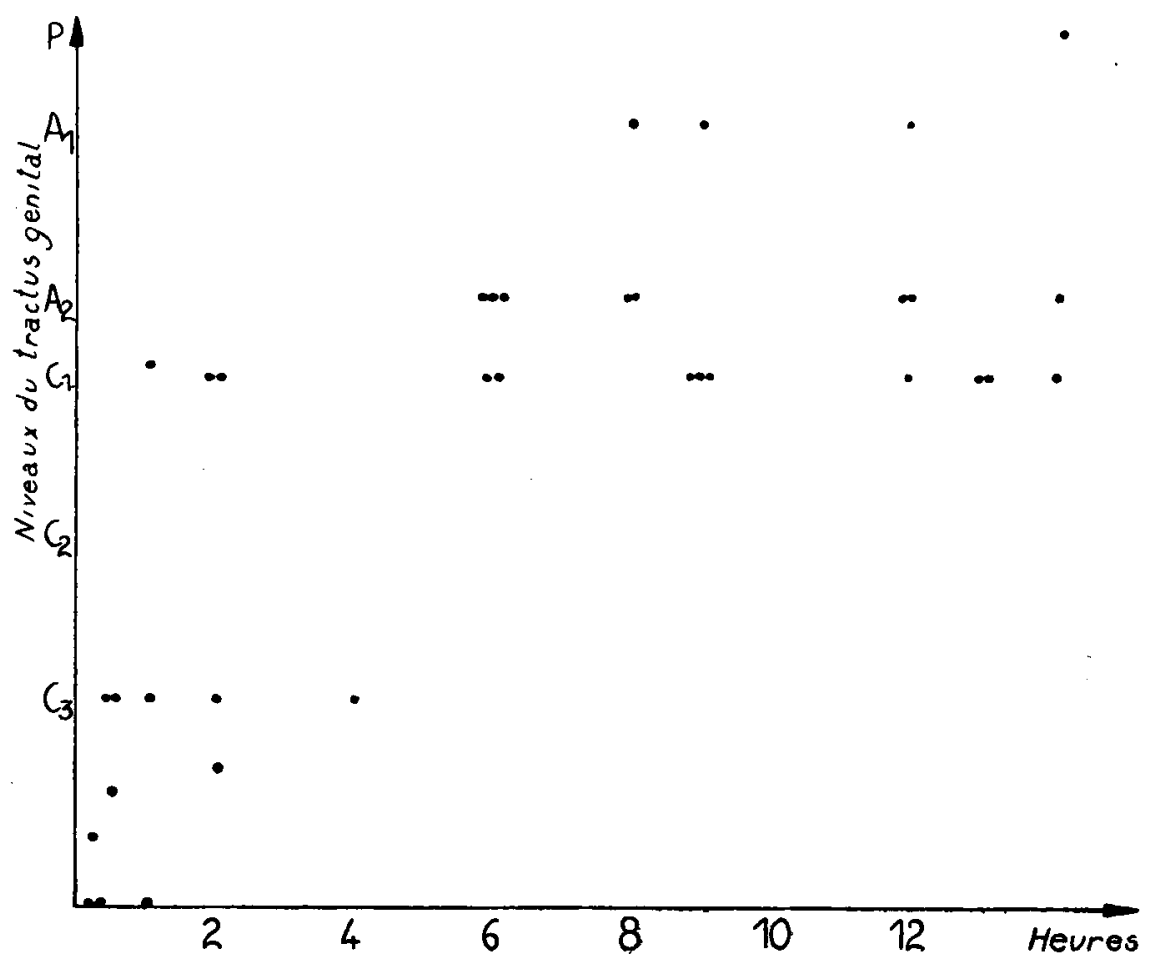

Frg. I. - Niveau du tractus génital atteint par les spermatozoïdes chez des Brebis accouplées dès l'apparition des chaleurs.

Sur les figures I et 2, l'abscisse représente les heures et l'ordonnée les longueurs parcourues par les spermatozoïdes (la longueur moyenne du tractus de l'extrémité postérieure du cervix au pavillon étant de $38 \mathrm{~cm}$ ).

On porte les valeurs qui indiquent le niveau atteint par les spermatozoïdes. La dispersion des points, pour les Brebis abattues en début et en fin de rut, montre une grande variabilité individuelle. C'est ainsi que les spermatozoïdes atteignirent le sommet des cornes utérines entre 9 et $\mathrm{r} 3$ heures après la copulation chez 5 Brebis accouplées dès l'apparition des chaleurs, et entre 9 et I2 heures chez 4 Brebis abattues vers la fin du rut.

Malgré cette grande variabilité, la corrélation entre les distances parcourues par les spermatozoïdes et le temps, est de 0,72 et 0,78 lorsque le coït a lieu 
en début et en fin de rut. Les coefficients de régression ont des valeurs significatives et égales $(I, 4)$, la régression n'étant toutefois pas linéaire. Les vitesses moyennes de remontée des spermatozoïdes qu'expriment ces coefficients sont

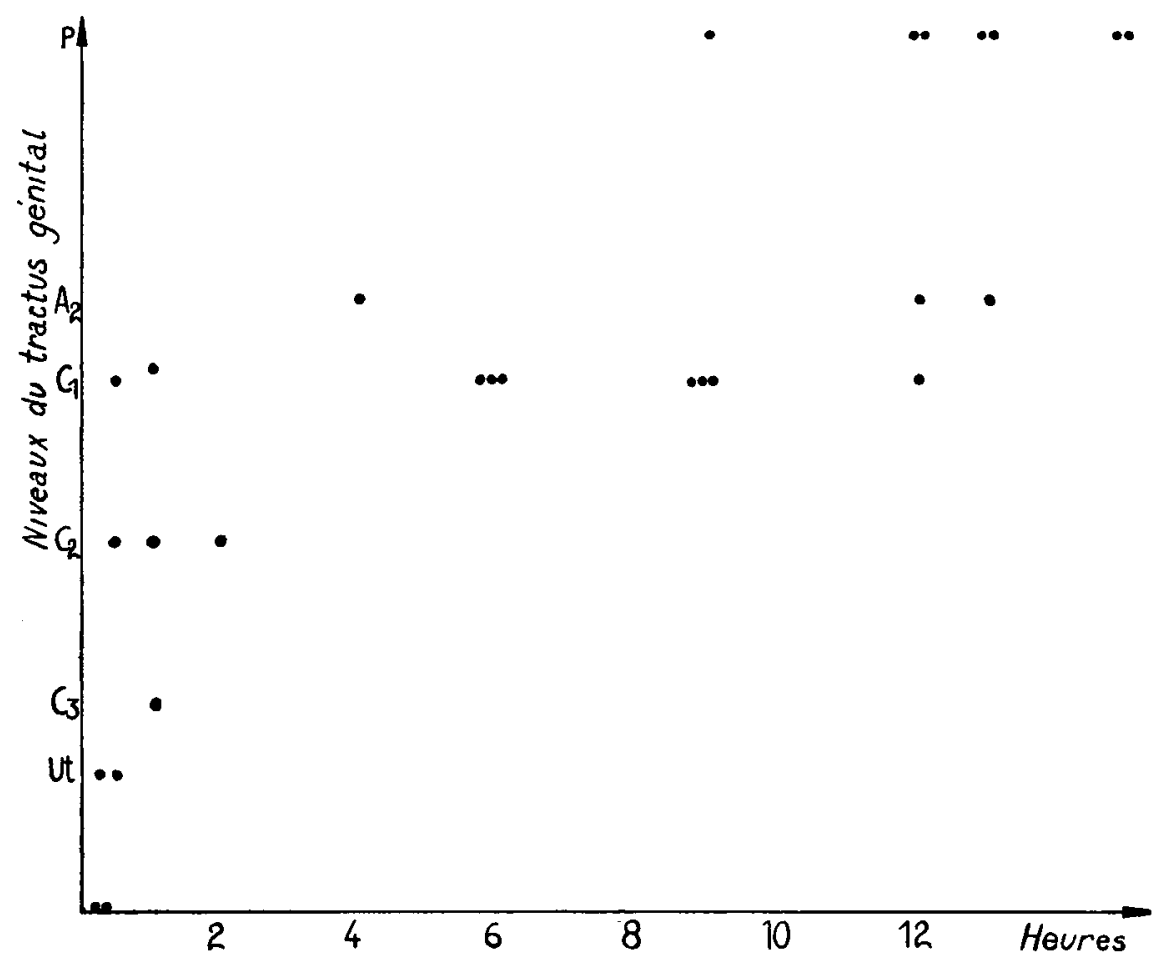

FıG. 2. - Niveau du tractus génital atteint par les spermatozoides chez des Brebis accouplées vers la fin des chaleurs.

donc indépendantes du moment des chaleurs. L'examen du col de l'utérus avant 1'accouplement nous a permis de constater que la remontée des gamètes mâles est plus rapide lorsque le cervix est largement ouvert. L'état du col de l'utérus pourrait donc faciliter, au départ, la progression des spermatozoïdes.

\section{NOMBRE DE SPERMATOZOIDES AUX DIFFERENTS NIVEAUX DU TRACTUS GENITAL}

La vitesse de progression des spermatozoïdes ne nous fournit aucune donnée quantitative ; or il est indispensable de connaître en fonction du temps le nombre de gamètes mâles présents aux différents niveaux du tractus génital.

D'après les tableaux I et II, il y a de très grandes variations relatives $d \mathbf{u}$ nombre de spermatozoïdes aux différents niveaux du tractus ; variations mises 
en évidence sur le graphique III où l'ordonnée exprime la racine carrée du nombre $(\mathrm{N})$ de spermatozoïdes trouvés dans la région du tractus génital indiquée en abscisses.

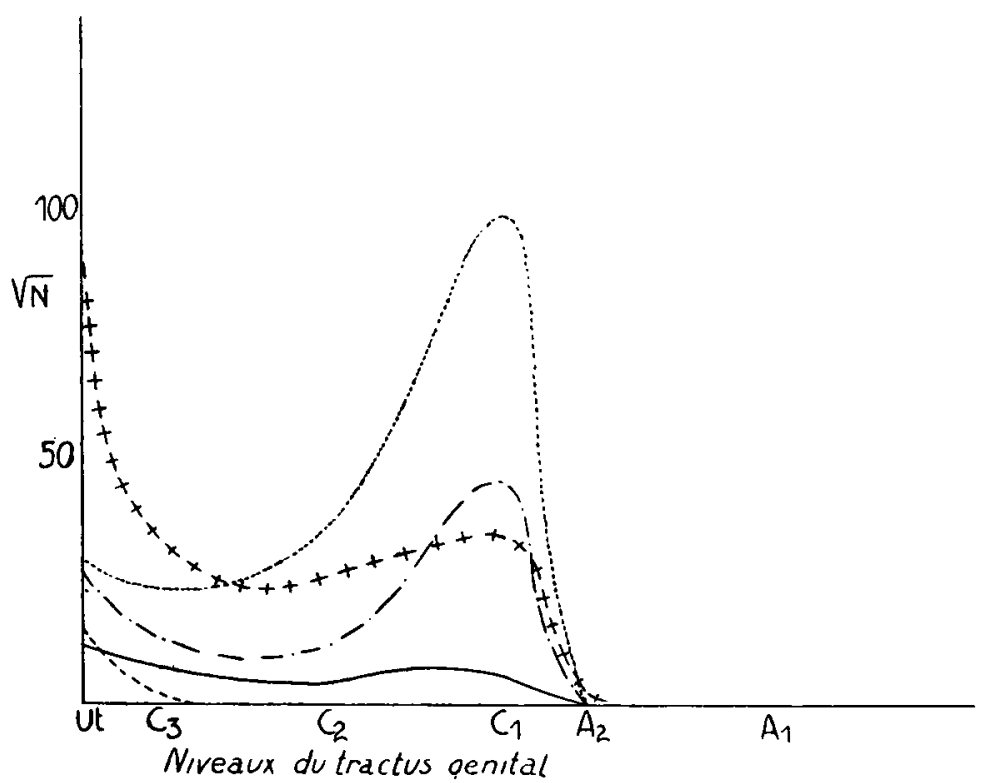

Fig. 3. - Racine carrée du nombre de spermatozoides $(\sqrt{\mathbb{N}})$. Courbes obtenues pour des temps de :

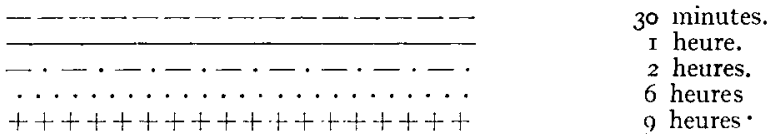

30 minutes après le coït, la plus grande quantité des spermatozoïdes se trouve dans l'utérus, leur nombre diminue en remontant vers le sommet des cornes. A une heure, on note la présence d'un nombre légèrement plus élevé de spermatozoïdes au sommet des cornes $\left(C_{1}\right)$, qu'aux autres niveaux utérins. A 2, 6, 9 et I2 heures, les courbes accusent un sommet en $C_{1}$, qui correspond à une accumulation de spermatozoïdes à l'extrémité supérieure des cornes utérines. La pente brusque de la courbe entre $\mathrm{C}_{1}$ et $\mathrm{A}_{2}$ (milieu de l'isthme) montre que seuls quelques spermatozoïdes progressent dans les trompes de Fallope.

Dans un autre ordre d'idées, le graphique IV prouve que le nombre total de spermatozoïdes présents dans l'ensemble des cornes, augmente jusqu'à I2 heures.

Néanmoins, les nombres de spermatozoïdes indiqués dans les tableaux I et II montrent qu'il ne pénètre dans l'utérus et les cornes, qu'un faible pourcentage de la multitude (de l'ordre du milliard) de spermatozoïdes éjaculés 
dans le vagin, compte tenu de la proportion de la surface utérine prélevée (environ le $\mathrm{I} / \mathrm{IOo}^{\mathrm{e}}$ de la surface totale). Le cervix limiterait donc le nombre total des spermatozoïdes qui remontent le tractus.

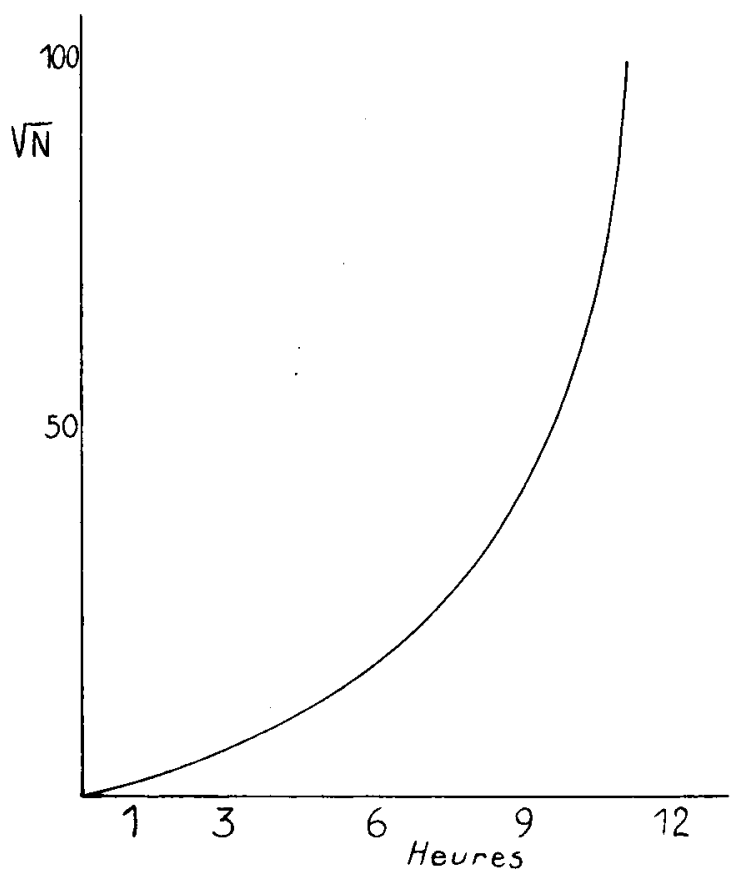

FiG. 4. - Variation de la racine carrée $(\sqrt{\mathrm{N}})$ du nombre total moyen de spermatozoides présents dans l'ensemble des cornes utérines, en fonction du temps.

L'état du cervix permettrait également d'expliquer, en partie, les variations, en valeur absolue, du nombre de spermatozoïdes trouvés en un même point du tractus chez des Brebis abattues à des temps comparables, variations qui apparaissent sur les tableaux I et II. On ne peut vraisemblablement imputer ces variations à la motilité du sperme et au pourcentage de spermatozoïdes anormaux, les contrôles de sperme effectués n'ayant pas révélé de différences appréciables entre les éjaculats (motilité $\geqslant 75 \%$, formes anormales $\leqslant 20 \%$ ).

\section{DISCUSSION}

Nos résultats sont en désaccord avec ceux de StaRkE et ceux de Schotr et PHILLIPS. Alors que ces derniers auteurs trouvent que les spermatozoïdes atteignent dans la plupart des cas le pavillon en 20 minutes, nous constatons en réalité une vitesse de remontée des spermatozoïdes beaucoup plus faible, et une grande variabilité individuelle. 
Nous avons comparé les nombres de spermatozoïdes trouvés par la méthode des frottis, à ceux obtenus à partir de coupes sériées longitudinales de fragments péalablement ligaturés aux deux extrémités. Les résultats identiques obtenus avec les deux techniques ont confirmé la valeur de la méthode des frottis. Les résultats de STARKE et de Schotr et Phillips sont peut-être dus à des fautes techniques. Des erreurs initiales nous avaient d'ailleurs montré qu'il fallait prendre de très grandes précautions dans le nettoyage du matériel. Nous avons ainsi été amenés à traiter les instruments et la verrerie au mélange sulfochromique et à ne les utiliser qu'une seule fois, au cours d'un prélèvement.

L'étude des variations du nombre de spermatozoïdes au niveau du tractus génital met en évidence le rôle important que joue le cervix dans la remontée des spermatozoïdes. Le col de l'utérus se comporte comme un lieu d'accumulation des spermatozoïdes. Il y a dans l'utérus une arrivée continuelle de spermatozoides ainsi que le prouvent d'une part l'augmentation, avec le temps, du nombre total moyen des spermatozoïdes dans les cornes utérines, d'autre part 1'accumulation des spermatozoïdes au sommet des cornes. Ces résultats sont à rapprocher de ceux obtenus par SkatKINE et RoumIantzewa chez la Lapine et, par GREEN chez la Brebis, qui signalent que le col de l'utérus jouerait le rôle d'un réservoir de spermatozoïdes, que ceux-ci quitteraient progressivement pour remonter le tractus.

En outre, le cervix constitue un obstacle à la progression des spermatozoïdes du vagin dans l'utérus, et limite le nombre de spermatozoïdes qui pénètrent plus avant dans le tractus ; c'est pourquoi son état de dilatation jouerait un rôle capital.

Une infime proportion des spermatozoïdes émis dans le vagin atteint le pavillon. La marge de sécurité, pour que la fécondation se produise, est donc faible, il nous est arrivé de ne trouver à 9 heures et à $I 2$ heures aucun spermatozoïde dans l'oviducte de Brebis ayant ovulé. Ceci explique ;certains cas de stérilité relative et justifie une étude des facteurs assurant cette remontée.

\section{CONCLUSIONS}

I $^{0}$ Nos recherches sur la vitesse de remontée des spermatozoïdes dans le tractus génital de la Brebis, nous ont permis de constater qu'il existe une grande variabilité individuelle. Toutefois, en aucun cas, les spermatozoïdes n'ont atteint la partie inférieure de l'oviducte avant 4 heures, et la partie supérieure avant 8 heures. Nous pouvons donc affirmer que les résultats de STARKE et de Schotr et PHILLips sont erronés.

$2^{\circ}$ Les coefficients de régression qui expriment la vitesse moyenne de remontée sont les mêmes en début et en fin de rut, l'équation de régression n'étant pas toutefois linéaire. Certes, les spermatozoïdes atteignent plus vite la partie supérieure des trompes pendant la fin de l'œstrus, mais cela tient au 
franchissement plus rapide d'un obstacle préliminaire : le col de l'utérus, dont le degré d'ouverture varie beaucoup.

$3^{\circ}$ Le nombre de spermatozoïdes présents dans les cornes utérines augmente pendant I2 heures; en outre, on constate une accumulation des spermatozoïdes au sommet des cornes utérines dès la première heure après le coït. Mais un très petit nombre de spermatozoïdes franchissent la jonction utérotubaire et atteignent la partie terminale de la trompe.

$$
\text { (Reçu pour publication le } 22 \text { février 1951). }
$$

\section{BIBLIOGRAPHIE}

BLANDAU (R. J.). - On the factors involved in sperm transport through the cervix uteri of the albino Rat. Amer. J. Anat., r945, r7\%, 253-72.

Blandau (R. J.), Jordane (I.), Money (W.L.), Ring (J. R.). - The rate of transport of spermatozoa in the Fallopian tubes of the Rat. Anal. Rec., I94I, 81, 83 .

Bland AU (R. J.), MoNEY (W. L.). - Observations on the rate of transport of spermatozoa in the female genital tract of the Rat. Anat. Rec., 1944, 90, 255-60.

Blandau (R. J.), ODOR (D. L.). - The total number of spermatozoa reaching various segments of the reproductive tract in the female albino Rat at intervals after insemination. Anat. Rec., 1949, 103, 93-го9.

Brewster (J. E.), MaY (R.), Cole (C. L.). - The time of ovulation and rate of spermatozoa travel in Cattle. Proc. Amer. Soc. Anim. Prod., I940, 33 rd Ann. Meet., 304-ro.

Evans (E. I.). - The transport of spermatozoa in the Dog. Amer. J. Physiol., 1933, 105, 287-93.

Ván Demark (N. L.), Moeller (A. N.). - Spermatozoa transport in the reproductive tract of Cow. J. Dairy Sci., I950, 33, 390-1.

FERIN (J.). - Facteurs hormonaux et migration des spermatozoïdes dans l'utérus chez la Femme. Ann. Endocrinol., 1948, 9, 77-83.

Green (W. W.), Winter (L. M.). - Studies on the physiology of reproduction in the Sheep. III : The time of ovulation and rate of sperm travel. Anat. Rec., I935, 61, 457-67.

Greenberg (B. E.), Nicholas (M. D.), Werthessen (Ph. D.), Berman (S.), Gargill (S. I.). - The transport of spermatozoa in the female reproductive tract. J. Urol., I945, 54, 57 r-4.

Hensen (V.). - Beobachtungen über die Befruchtung und Entwicklung des Kaninchens und Meerschwinchens. Zeitschr. f. Anat. u. Entwick., I876, 1, 213-73.

Kelley (R. B.). - Studies in fertility of Sheep. Bull. Counc. Sci. Industr. Res. Austr., $1937, \mathrm{n}^{\circ} 112$.

Lopyrin (A. I.), Logrnova (N. V.). - Vitesse de remontée et temps de survie des spermatozoïdes dans le tractus génital de la Brebis. Sovetsk. Zootech., r939, $n^{\circ}$ 2-3, 144-9.

Mimura (H.). - Studies on the mechanism of travel of spermatozoa through the oviduct in the domestic Fowl. Dept. Agric. Kyusyu Imp. Univ., I94r, 6, 168-259.

Parker (G. H.). - - Passage of sperms and eggs through oviducts of Rabbit and of human being with consideration of Sampson's theory of hemorragic of chocolate cysts. Amer. J. Obstr. Gynec., 1932, 23, 619-26.

Philmips (R. W.), Andrews (F. N.). -- The speed of travel of Ram spermatozoa. Anat. Rec., I937, 68, I27-32.

ODOR (L.). - - The number of spermatozoa reaching various segments of the oviduct of the Rat, I2, 24 and 36 hours after mating. Anat. Rec., I948, 100, 733.

Quinlan (J.), MARE (G. S.), Roux (L. L.). - The vitality of the spermatozoa in the genital tract of the Merino Ewe, with special reference to its practical application in breeding. I8th Rep. Direction Vet. Serv. Anim. Industr. Union of South Africa, I 932, 2, 83 I-87o.

SchotT (R. G.), Phillips (R. W.). - Rate of sperm travel and time of ovulation in Sheep. Anat. Rec., 1941, 79, 53r-40.

Skatkine (P. N.), Roumiantzewa (E. J.). - Vitesse de déplacement des spermatozoïdes dans les organes sexuels femelles. Rap. Acad. Sov. Len. Sci. Agron., r948, 10, 45-51.

STARKE (N. C.). - The sperm picture of Rams of different breeds as an indication of their fertility. II. The rate of sperm travel in the genital tract of the Ewe. Onderst. J. Vet. Sci. An. Ind., I949, 22, 415-525. 